\title{
Optimized SFCW Radar Sensor Aiming at Fall Detection in a Real Room Environment
}

\author{
Marco Mercuri $^{1}$, Ping Jack Soh ${ }^{1,2}$, Luigi Boccia ${ }^{3}$, Dominique Schreurs ${ }^{1}$, Guy A. E. Vandenbosch ${ }^{1}$, \\ Paul Leroux ${ }^{4,5}$, and Giandomenico Amendola ${ }^{3}$ \\ ${ }^{1}$ KU Leuven, Div. ESAT-TELEMIC, Leuven, Belgium \\ ${ }^{2}$ Universiti Malaysia Perlis, School of Computer \& Communication Engineering, Perlis, Malaysia \\ ${ }^{3}$ UNICAL, Div. DEIS-MICROWAVELAB, Arcavacata di Rende, Italy \\ ${ }^{4}$ KU Leuven, Div. ESAT-MICAS, Leuven, Belgium \\ ${ }^{5}$ K.H.Kempen, Div. IBW-RELIC, Geel, Belgium
}

\begin{abstract}
An optimization of a Stepped-Frequency Continuous Wave (SFCW) radar for contactless fall detection and tag-less localization is presented and discussed. Wideband antennas with different topologies are investigated to solve the backscattering problem, which strongly overwhelms the signal when the radar is operated on a wall. Experimental evaluations with a real human subject indicated accurate detection of the target's absolute distance and speed signal in a typical room setting.

Index Terms - Fall detection, health monitoring, radar remote sensing, SFCW radar, tag-less localization.
\end{abstract}

\section{INTRODUCTION}

Innovative technology approaches in healthcare are increasingly investigated as triggered by the rapid and continual growth of the elderly people globally. In particular, the demand for reliable in-door monitoring systems is a core focus [1]. Injuries sustained from fall incidents at home are considered the most dangerous cause of fatal accidents for elderly people, and it also represents the third cause of chronic disability [2]. Instant detection of a fall event significantly reduces the mortality risk, and in turn increases the chance of the person's return to independent living. As opposed to body-worn solutions (e.g., accelerometers), remote detection of fall offers the fundamental advantage that no action by the elderly is required. Systems under investigation are based on video cameras, floor vibration, and acoustic sensors [3]. However, due to the unacceptable high number of reported 'false positives', these approaches are not suitable to work under real conditions. An alternative approach based on the use of radar techniques was proposed by the authors [4]. The paper demonstrated the feasibility of using a Stepped-Frequency Continuous Wave (SFCW) radar for remote fall detection. The experimental results were obtained by using non-optimized hardware. In this paper, we present an improved SFCW radar, optimized for practical circumstances. In particular, we analyse and discuss the challenges in the antenna design to allow for SFCW radar operation in a realistic room setting.

The original experimental results in [4] made use of bulky horn antennas. Due to their inherent high directivity, the radar sensor was only able to detect movements when the target was in the antennas' line of sight (LoS) paths. The optimized system presented in this paper is able to cover the whole area of a typical $\left(5 \times 5 \mathrm{~m}^{2}\right)$ room, usually available in a private home. The human subject is now also allowed free movement. Moreover, the large volume of horn antennas is not practical for real applications. A planar antenna solution that can be integrated with the radar electronics in a package is preferred for practical applications. The wide bandwidth, related to SFCW radar operation, combined with the real room setting poses design challenges. In particular, the problem of backscattering is fundamental as the radar sensor is to be fixed on a wall or ceiling. To this end, three wideband antennas have been designed, and are evaluated and compared in terms of backscattering.

In Section II the optimized SFCW radar sensor is reported. The antenna designs are presented in Section III, and the experimental results are discussed in Section IV.

\section{SFCW RADAR SENSOR}

The block diagram of the radar sensor is shown in Fig. 1. The SFCW waveform is generated by a fractional-N PLL with integrated VCO whose synthesizer is properly programmed by a microcontroller. The same microcontroller also acquires the measured $\mathrm{I}$ and $\mathrm{Q}$ baseband components. This represents a notable improvement in terms of portability compared to the radar reported in [4], where a bulky and complex signal generator was used, as well as an oscilloscope for the data acquisition.

In order to cover a range of $5 \mathrm{~m}$, the frequency step $\Delta f$ has been fixed to $30 \mathrm{MHz}$ while the number of steps $N$ has 
been chosen to be equal to 34 in order to obtain a range resolution of about $15 \mathrm{~cm}$. The total band $N \cdot \Delta f$ is 1.02 $\mathrm{GHz}$ positioned between $1-1.99 \mathrm{GHz}$. The time interval $T$ is $100 \mu$ s while the pulse dwell time is about $60 \mu$ s. The burst interval $N \cdot T$ is $3.4 \mathrm{~ms}$, which satisfies the assumption that the target is static during each burst interval. The frequency used to detect the speed is $1 \mathrm{GHz}$, the lower limit of the burst's frequency range. Finally, the transmit power level feeding the antenna is $4 \mathrm{dBm}$.

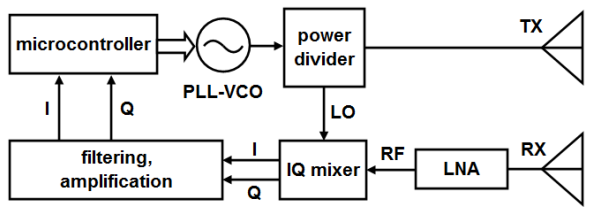

Fig. 1. Radar sensor architecture.

\section{ANTENNA DESIGN}

\section{A. Design Challenges}

The radar system operation between 1.00 and $1.99 \mathrm{GHz}$ translates into an antenna impedance bandwidth of c.a. $68 \%$, which falls under the "Ultra-wide band (UWB)" category. Whereas many publications have reported on UWB antenna designs, they are not automatically applicable to the radar sensor application.

Two particular challenges are crosstalk and backscattering. As crosstalk can be decreased by positioning the antennas further apart, this paper mainly concentrates on backscattering. As the radar sensor is to be fixed on a wall or ceiling, strong signal reflections will overwhelm the entire collected target information and saturate the low noise amplifier (LNA), besides dissipating valuable transmitted power. The presence of such clutter in the measured data especially renders the data processing for the positioning and speed detection difficult.

In the next section, the design of three planar antennas with different topologies is reported. They are chosen intentionally to enable the assessment of relatively distinct radiation patterns, in order to determine the best topology for this radar's application.

\section{B. Antenna Designs}

The first antenna design is a semi-elliptical monopole antenna slotted with circular and semi-circular slots. The overall substrate size is $65 \times 104 \mathrm{~mm}^{2}$, with its reverse side printed with a partial ground plane. These slots and ground plane structures are introduced to enable bandwidth broadening, with a simultaneous size reduction. This antenna is printed on a Taconic TLY-5 substrate with relative permittivity, $\varepsilon \mathrm{r}=2.2$, loss tangent, $\tan \delta=0.0009$ and substrate height, $\mathrm{h}=0.51 \mathrm{~mm}$.
The second antenna is a Vivaldi radiator designed to operate within the same frequency range. In general, Vivaldi antennas are used to enable broad impedance bandwidth, as it features a shaped radiator-end transition. It is sized at $90 \times 120 \mathrm{~mm}^{2}$, and printed on two Arlon AR350 (with $\varepsilon_{r}=3.58$, $\tan \delta=0.026$ ) substrates, with the height of each layer, $h=0.8 \mathrm{~mm}$. Their reverse sides stacked against each other, resulting in a combined substrate height, $h=1.6 \mathrm{~mm}$. A $50 \Omega$ feed line is sandwiched between these two layers. Due to its special topology, an end-fire radiation is produced.

The third antenna is designed based on a bow-tie topology. An inverse metallic-substrate structure on the Arlon AR350 is used to realize a magnetic bow-tie radiator instead of the typically-used electric bow-tie. This radiator was fed using through a CPW transmission line and an SMA connector. This antenna is slightly larger, sized at $90 \times 145 \mathrm{~mm}^{2}$, fed using a co-planar waveguide (CPW) line with $3.5 \mathrm{~mm}$ gap on either side of a $4 \mathrm{~mm}$ centre feed line. With the intent to limit the backscattering effect, the design of this antenna has been vastly modified to ensure the least backwards radiation. To this end, the antenna has been designed with a ground plane at a $\lambda / 2$ from the radiating layer. The interlayer distance was filled with a $44 \mathrm{~mm}$ thick foam spacer. All three structures are shown in Fig. 2.

The experimental results of the designed antennas are presented in next Section.

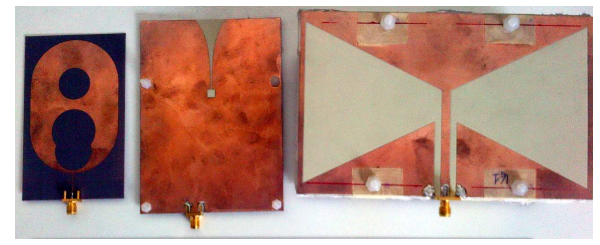

Fig. 2. Fabricated prototypes of the three evaluated antennas.

\section{EXPERIMENTAL RESULTS}

\section{A. Antenna Results}

First, the fabricated antennas have been characterized before testing them in the radar sensor set-up. In order to determine their bandwidth, the reflection coefficient (S11) has been measured with a vector network analyzer. The bow tie antenna generated the largest $-10 \mathrm{~dB}$ impedance bandwidth $(768 \mathrm{MHz})$, followed by the semi-elliptical antenna (746 MHz), and the Vivaldi antenna (638 MHz).

Next, radiation patterns measurements have been carried out in an anechoic chamber. Their measured normalized patterns at $1.5 \mathrm{GHz}$ are shown in Fig. 3. All three antennas indicated significant backwards radiation on their respective E-planes. On the H-plane, however, a more 
directive main (forward) and back lobe is observed for the bow tie antenna compared to the other two, indicating a bidirectional radiation. This resulted in a narrow half-power beam-width (HPBW) of $58^{\circ}$, between $\pm 29^{\circ}$. On the contrary, the Vivaldi antenna indicated the largest forward coverage area, between $-60^{\circ}$ and $+165^{\circ}$, i.e., a total of $225^{\circ}$. This implies its suitability when used for a wider room area, or being mounted on a lower ceiling, at the expense of a lower directivity.

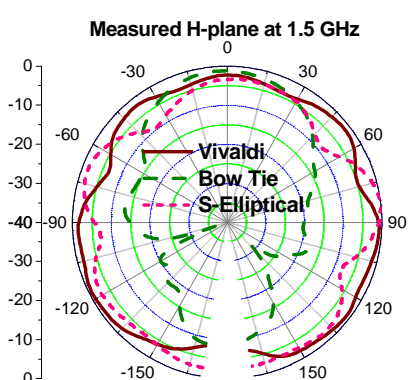

(a)

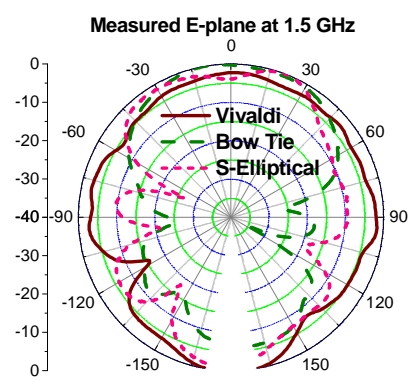

(b)
Fig. 3. Measured normalized E- and H-planes patterns for all three antennas at $1.5 \mathrm{GHz}$ center operating frequency.

\section{B. Radar Sensor Results}

The overall radar system evaluation is performed by positioning the radar sensor at a fixed $1.5 \mathrm{~m}$ height. A standard, semi-elliptical antenna has been used as the transmitting antenna, while the tested antennas are mounted on the receiving side of the radar. The reciprocal antenna radiation property renders this assumption valid, as long as the tested antennas are consistently mounted at either the radar's receiving or transmitting end. To avoid transmit-receive signal cross-coupling, the two antennas are spaced by a distance of $20 \mathrm{~cm}$, which is at a full $\lambda$ distance of the $1.5 \mathrm{GHz}$ center operating wavelength. A 50 $\mathrm{mm}$ thick broadband microwave absorber is also placed in between them to ensure that this cross-coupling factor does not invalidate the investigation of antenna back scattering effect.

Measurements for target's position have been conducted with the person standing at four different absolute distances from the radar, while moving from $5 \mathrm{~m}$ to $1 \mathrm{~m}$ towards the antenna for speed detection. The same conditions have been applied for each of the three receiving antennas. Due to the elevated backscattering, the semi-elliptical antenna is not able to detect neither the absolute distances nor the speed of the target in the whole room. In fact, the much stronger contribution of the backscattering is added to the weaker reflection from the target. This involves decreasing the gain of the baseband amplifiers in the sensor hardware to avoid the saturation of the analog to digital converters (ADCs). The longer is the distance, the weaker is the reflection of the target such that it becomes no longer perceptible by the ADCs' resolution. The same problem, though less emphasized, has been experienced for the Vivaldi antenna. In fact, the target has been detected only when present in the half of the room facing the radar. On the contrary, the best results have been obtained with the bow-tie antenna. In fact, due to its limited backscattering, it was possible to increase the gain of the baseband amplifiers and then being able to cover the full room. The obtained results, regarding both absolute distance and speed detection, are shown in Table I.

TABLE I

TARGET DETECTION RESULTS

\begin{tabular}{|c|c|c|c|c|}
\hline \multirow{2}{*}{ Antenna Type } & \multicolumn{4}{|c|}{ Target detectable vs. absolute } \\
\cline { 2 - 5 } & $1 \mathrm{~m}$ & $2 \mathrm{~m}$ & $3 \mathrm{~m}$ & $4 \mathrm{~m}$ \\
\hline Semi-elliptical & YES & NO & NO & NO \\
\hline Vivaldi & YES & YES & NO & NO \\
\hline Bow-tie & YES & YES & YES & YES \\
\hline
\end{tabular}

\section{CONCLUSION}

An optimized SFCW radar sensor aiming at fall detection and tag-less localization in a realistic room setting has been presented. Besides compacting the electronics hardware, particular focus has been on the backscattering issue in the antenna part. Three types of wideband antennas have been designed and tested. Results show that the bow-tie antenna represents the most suitable antenna type for the SFCW radar aiming to determine absolute distance and speed signal of the person.

\section{ACKNOWLEDGEMENTS}

This work has been supported by FWO-Flanders, KU Leuven GOA project and the Malaysian Ministry of Higher Education (MOHE).

\section{REFERENCES}

[1] O. Boric-Lubecke and V.M. Lubecke, "Wireless house calls: using communications technology for health care and monitoring," IEEE Microwave Magazine, vol. 3, pp. 43-48, Sep. 2002.

[2] S. R. Lord, C. Sherrington, and H. B. Menz, Falls in Older People: Risk Factors and Strategies for Prevention, Cambridge, U.K.: Cambridge Univ. Press, 2007.

[3] M. Yu, S. M. Naqvi, and J. Chambers, "A robust fall detection system for elderly in a smart room," International Conference on Acoustics Speech and Signal Processing, pp. 1666 -1669, March 2010.

[4] M. Mercuri, D. Schreurs, and P. Leroux, "SFCW microwave radar for in-door fall detection," IEEE Topical Conference on Biomedical Wireless Technologies, Networks, and Sensing Systems (BioWireleSS), pp. 53-56, 15-18 Jan. 2012. 